\title{
On the Paranormed Nörlund Sequence Space of Nonabsolute Type
}

\author{
Medine Yeşilkayagil ${ }^{1}$ and Feyzi Başar ${ }^{2}$ \\ ${ }^{1}$ Department of Mathematics, Uşak University, 1 Eylül Campus, 64200 Uşak, Turkey \\ ${ }^{2}$ Department of Mathematics, Fatih University, Hadımköy Campus, Büyükçekmece, 34500 İstanbul, Turkey
}

Correspondence should be addressed to Feyzi Başar; feyzibasar@gmail.com

Received 3 December 2013; Accepted 2 February 2014; Published 26 March 2014

Academic Editor: M. Mursaleen

Copyright ( 2014 M. Yeşilkayagil and F. Başar. This is an open access article distributed under the Creative Commons Attribution License, which permits unrestricted use, distribution, and reproduction in any medium, provided the original work is properly cited.

\begin{abstract}
Maddox defined the space $\ell(p)$ of the sequences $x=\left(x_{k}\right)$ such that $\sum_{k=0}^{\infty}\left|x_{k}\right|^{p_{k}}<\infty$, in Maddox, 1967. In the present paper, the Nörlund sequence space $N^{t}(p)$ of nonabsolute type is introduced and proved that the spaces $N^{t}(p)$ and $\ell(p)$ are linearly isomorphic. Besides this, the alpha-, beta-, and gamma-duals of the space $N^{t}(p)$ are computed and the basis of the space $N^{t}(p)$ is constructed. The classes $\left(N^{t}(p): \mu\right)$ and $\left(\mu: N^{t}(p)\right)$ of infinite matrices are characterized. Finally, some geometric properties of the space $N^{t}(p)$ are investigated.
\end{abstract}

\section{Introduction}

We denote the space of all sequences of complex entries by $\omega$. Any vector subspace of $\omega$ is called a sequence space. We write $\ell_{\infty}, c$, and $c_{0}$ for the spaces of all bounded, convergent, and null sequences, respectively. Also by $b s, c s, \ell_{1}$, and $\ell_{p}$, we denote the spaces of all bounded, convergent, absolutely and $p$-absolutely convergent series, respectively.

A linear topological space $X$ over the real field $\mathbb{R}$ is said to be a paranormed space if there is a subadditive function $g: X \rightarrow \mathbb{R}$ such that $g(\theta)=0, g(x)=g(-x)$ and scalar multiplication is continuous; that is, $\left|\alpha_{n}-\alpha\right| \rightarrow 0$ and $g\left(x_{n}-x\right) \rightarrow 0$ imply $g\left(\alpha_{n} x_{n}-\alpha x\right) \rightarrow 0$ for all $\alpha$ 's in $\mathbb{R}$ and all $x$ 's in $X$, where $\theta$ is the zero vector in the linear space $X$. Assume here and after that $\left(p_{k}\right)$ is a bounded sequence of strictly positive real numbers with sup $p_{k}=H$ and $M=\max \{1, H\}$. Then, the linear spaces $\ell(p)$ and $\ell_{\infty}(p)$ were defined by Maddox in [1] (see also [2,3]) as follows:

$$
\begin{array}{r}
\ell(p)=\left\{x=\left(x_{k}\right) \in \omega: \sum_{k}\left|x_{k}\right|^{p_{k}}<\infty\right\} \\
\text { with } 0<p_{k} \leq H<\infty,
\end{array}
$$

$$
\ell_{\infty}(p)=\left\{x=\left(x_{k}\right) \in \omega: \sup _{k \in \mathbb{N}}\left|x_{k}\right|^{p_{k}}<\infty\right\}
$$

which are the complete spaces paranormed by

$$
\begin{aligned}
& g_{1}(x)=\left(\sum_{k}\left|x_{k}\right|^{p_{k}}\right)^{1 / M}, \\
& g_{2}(x)=\sup _{k \in \mathbb{N}}\left|x_{k}\right|^{p_{k} / M} \text { iff } \inf _{k \in \mathbb{N}} p_{k}>0,
\end{aligned}
$$

respectively, where $\mathbb{N}=\{0,1,2, \ldots\}$. For simplicity in notation, here and in what follows, the summation without limits runs from 0 to $\infty$. We assume throughout that $p_{k}^{-1}+$ $\left(p_{k}^{\prime}\right)^{-1}=1$, provided $1<\inf p_{k} \leq H<\infty$, and denote the collection of all finite subsets of $\mathbb{N}$ by $\mathscr{F}$.

For the sequence spaces $\lambda$ and $\mu$, define the set $S(\lambda, \mu)$ by

$$
S(\lambda, \mu)=\left\{z=\left(z_{k}\right) \in \omega: x z=\left(x_{k} z_{k}\right) \in \mu \forall x \in \lambda\right\} .
$$


With the notation of (3), the alpha-, beta-, and gamma-duals of a sequence space $\lambda$, which are, respectively, denoted by $\lambda^{\alpha}$, $\lambda^{\beta}$, and $\lambda^{\gamma}$, are defined by

$$
\lambda^{\alpha}=S\left(\lambda, \ell_{1}\right), \quad \lambda^{\beta}=S(\lambda, c s), \quad \lambda^{\gamma}=S(\lambda, b s) .
$$

If a sequence space $\lambda$ paranormed by $g$ contains a sequence $\left(b_{n}\right)$ with the property that, for every $x \in \lambda$, there is a unique sequence of scalars $\left(\alpha_{n}\right)$ such that

$$
\lim _{n \rightarrow \infty} g\left(x-\sum_{k=0}^{n} \alpha_{k} b_{k}\right)=0,
$$

then $\left(b_{n}\right)$ is called a Schauder basis (or briefly basis) for $\lambda$. The series $\sum_{k} \alpha_{k} b_{k}$ which has the sum $x$ is then called the expansion of $x$ with respect to $\left(b_{n}\right)$ and written as $x=\sum_{k} \alpha_{k} b_{k}$.

Let $\lambda, \mu$ be any two sequence spaces, and let $A=\left(a_{n k}\right)$ be an infinite matrix of complex numbers $a_{n k}$, where $k, n \in \mathbb{N}$. Then, we say that $A$ defines a matrix transformation from $\lambda$ into $\mu$ and we denote it by writing $A: \lambda \rightarrow \mu$, if for every sequence $x=\left(x_{k}\right) \in \lambda$, the sequence $A x=\left\{(A x)_{n}\right\}$, the $A$ transform of $x$, is in $\mu$, where

$$
(A x)_{n}=\sum_{k} a_{n k} x_{k} \quad \text { for each } n \in \mathbb{N} .
$$

By $(\lambda: \mu)$, we denote the class of all matrices $A$ such that $A: \lambda \rightarrow \mu$. Thus, $A \in(\lambda: \mu)$ if and only if the series on the right side of (6) converges for each $n \in \mathbb{N}$ and every $x \in \lambda$, and we have $A x \in \mu$ for all $x \in \lambda$. Also, we write $A_{n}=\left(a_{n k}\right)_{k \in \mathbb{N}}$ for the sequence in the $n$th row of $A$.

Now, following Peyerimhoff [4, pp. 17-19] and Mears [5], we give short knowledge on the Nörlund means. Let $\left(t_{k}\right)$ be a sequence of nonnegative real numbers with $t_{0}>0$ and write $T_{n}=\sum_{k=0}^{n} t_{k}$ for all $n \in \mathbb{N}$. Then, the Nörlund means with respect to the sequence $t=\left(t_{k}\right)$ is defined by the matrix $N^{t}=$ $\left(a_{n k}^{t}\right)$ which is given by

$$
a_{n k}^{t}= \begin{cases}\frac{t_{n-k}}{T_{n}}, & 0 \leq k \leq n \\ 0, & k>n\end{cases}
$$

for all $k, n \in \mathbb{N}$. It is known that the Nörlund matrix $N^{t}$ is a Toeplitz matrix if and only if $t_{n} / T_{n} \rightarrow 0$, as $n \rightarrow \infty$, and is reduced in the case $t=e=(1,1,1, \ldots)$ to the matrix $C_{1}$ of arithmetic means. Additionally, for $t_{n}=A_{n}^{r-1}$ for all $n \in \mathbb{N}$, the method $N^{t}$ is reduced to the Cesàro method $C_{r}$ of order $r>-1$, where

$$
A_{n}^{r}= \begin{cases}\frac{(r+1)(r+2) \cdots(r+n)}{n !}, & n=1,2,3, \ldots, \\ 1, & n=0 .\end{cases}
$$

Let $t_{0}=D_{0}=1$ and define $D_{n}$ for $n \in\{1,2,3, \ldots\}$ by

$$
D_{n}=\left|\begin{array}{cccccc}
t_{1} & 1 & 0 & 0 & \cdots & 0 \\
t_{2} & t_{1} & 1 & 0 & \cdots & 0 \\
t_{3} & t_{2} & t_{1} & 1 & \cdots & 0 \\
\vdots & \vdots & \vdots & \vdots & \ddots & \vdots \\
t_{n-1} & t_{n-2} & t_{n-3} & t_{n-4} & \cdots & 1 \\
t_{n} & t_{n-1} & t_{n-2} & t_{n-3} & \cdots & t_{1}
\end{array}\right| .
$$

The inverse matrix $U^{t}=\left(u_{n k}^{t}\right)$ of the matrix $N^{t}=\left(a_{n k}^{t}\right)$ is given by Mears in [5] as follows:

$$
u_{n k}^{t}= \begin{cases}(-1)^{n-k} D_{n-k} T_{k}, & 0 \leq k \leq n, \\ 0, & k>n\end{cases}
$$

for all $k, n \in \mathbb{N}$. Also, one can derive by straightforward calculation for all $k \in\{1,2,3, \ldots\}$ that

$$
D_{k}=\sum_{j=1}^{k-1}(-1)^{j-1} t_{j} D_{k-j}+(-1)^{k-1} t_{k}
$$

The rest of this paper is organized as follows.

In Section 2, the complete paranormed Nörlund sequence space $N^{t}(p)$ is introduced and proved that $N^{t}(p)$ is linearly isomorphic to the space $\ell(p)$ and the basis for the space $N^{t}(p)$ is determined. Section 3 is devoted to the alpha-, beta-, and gamma-duals of the space $N^{t}(p)$. In Section 4 , the classes $\left(N^{t}(p): \mu\right)$ and $\left(\mu: N^{t}(p)\right)$ of infinite matrices are characterized, where $\mu$ denotes any given sequence space. In Section 5 , the rotundity of the space $N^{t}(p)$ is characterized and some results related to this concept are given. In the final section of the paper, the significance of the space is mentioned and further suggestions are recorded.

\section{The Nörlund Sequence Space $N^{t}(p)$ of Nonabsolute Type}

In this section, we define the Nörlund sequence space $N^{t}(p)$ and prove that $N^{t}(p)$ is linearly isomorphic to the space $\ell(p)$, where $0<p_{k} \leq H<\infty$ for all $k \in \mathbb{N}$. Finally, we give the basis for the space $N^{t}(p)$.

Let $\lambda$ be any sequence space. Then, the matrix domain $\lambda_{A}$ of an infinite matrix $A$ in $\lambda$ is defined by

$$
\lambda_{A}=\left\{x=\left(x_{k}\right) \in \omega: A x \in \lambda\right\} .
$$

In [6], Choudhary and Mishra defined the sequence space $\overline{\ell(p)}$ which consists of all sequences such that $B$-transforms of them are in $\ell(p)$, where $B=\left(b_{n k}\right)$ is defined by

$$
b_{n k}= \begin{cases}1, & 0 \leq k \leq n \\ 0, & k>n\end{cases}
$$

Başar and Altay [7] examined the space $b s(p)$ which was formerly defined by Başar [8] as the set of all series whose sequences of partial sums are in the space $\ell_{\infty}(p)$. With the notation of (12), the spaces $\overline{\ell(p)}$ and $b s(p)$ can be redefined by

$$
\overline{\ell(p)}=[\ell(p)]_{B}, \quad b s(p)=\left[\ell_{\infty}(p)\right]_{B} .
$$

In [9], Başar and Altay defined the sequence space $r^{q}(p)$ which consists of all sequences such that $R^{q}$-transforms of 
them are in $\ell(p)$, where $R^{q}=\left(r_{n k}^{q}\right)$ is the matrix of Riesz mean; that is,

$$
r^{q}(p)=\{\ell(p)\}_{R^{q}}, \quad r_{p}^{q}=\left(\ell_{p}\right)_{R^{q}} .
$$

In [10], Wang defined the sequence space $X_{a(p)}$ consisting of all sequences whose $N^{t}$-transforms are in $\ell_{p}$ which is a Banach space with the norm

$$
\|x\|_{p}=\left(\sum_{k}\left|\frac{1}{T_{k}} \sum_{j=0}^{k} t_{k-j} x_{j}\right|^{p}\right)^{1 / p} \quad \text { with } 1 \leq p<\infty .
$$

Now, we introduce the Nörlund sequence space $N^{t}(p)$ defined by

$$
\begin{array}{r}
N^{t}(p):=\left\{x=\left(x_{k}\right) \in \omega: \sum_{k}\left|\frac{1}{T_{k}} \sum_{j=0}^{k} t_{k-j} x_{j}\right|^{p_{k}}<\infty\right\} \\
\text { with } 0<p_{k} \leq H<\infty .
\end{array}
$$

It is natural that the space $N^{t}(p)$ can also be defined with the notation of (12) that $N^{t}(p)=\{\ell(p)\}_{N^{t}}$.

Define the sequence $y=\left(y_{k}\right)$ by the $N^{t}$-transform of a sequence $x=\left(x_{k}\right)$; that is,

$$
y_{k}=\frac{1}{T_{k}} \sum_{j=0}^{k} t_{k-j} x_{j} \quad \forall k \in \mathbb{N} .
$$

Theorem 1. $N^{t}(p)$ is a complete linear metric space paranormed by $g$ defined by

$$
g(x)=\left(\sum_{k}\left|\frac{1}{T_{k}} \sum_{j=0}^{k} t_{k-j} x_{j}\right|^{p_{k}}\right)^{1 / M} \quad \text { with } 0<p_{k} \leq H<\infty .
$$

Proof. Since this can be shown by a routine verification, we omit the detail.

Remark 2. One can easily see that the absolute property does not hold on the space $N^{t}(p)$; that is, $g(x) \neq g(|x|)$ for at least one sequence in the space $N^{t}(p)$, and this says that $N^{t}(p)$ is a sequence space of nonabsolute type, where $|x|=\left(\left|x_{k}\right|\right)$.

Theorem 3. The Nörlund sequence space $N^{t}(p)$ of nonabsolute type is linearly isomorphic to the space $\ell(p)$, where $0<p_{k} \leq$ $H<\infty$ for all $k \in \mathbb{N}$.

Proof. To prove the theorem, we should show the existence of a linear bijection between the spaces $N^{t}(p)$ and $\ell(p)$ for $0<p_{k} \leq H<\infty$. Consider the transformation $T$ defined, with the notation of (18), from $N^{t}(p)$ to $\ell(p)$ by $x \mapsto y=$ $T x=N^{t} x$. The linearity of $T$ is clear. Further, it is trivial that $x=\theta$ whenever $T x=\theta$ and hence $T$ is injective. by

Let us take any $y \in \ell(p)$ and define the sequence $x=\left(x_{k}\right)$

$$
x_{k}=\sum_{i=0}^{k}(-1)^{k-i} D_{k-i} T_{i} y_{i} \quad \forall k \in \mathbb{N} .
$$

Therefore, we see from (19) that

$$
\begin{aligned}
g(x) & =\left(\sum_{k}\left|\frac{1}{T_{k}} \sum_{j=0}^{k} t_{k-j} x_{j}\right|^{p_{k}}\right)^{1 / M} \\
& =\left(\sum_{k}\left|\frac{1}{T_{k}} \sum_{j=0}^{k} t_{k-j} \sum_{i=0}^{j}(-1)^{j-i} D_{j-i} T_{i} y_{i}\right|^{p_{k}}\right)^{1 / M} \\
& =\left(\sum_{k}\left|y_{k}\right|^{p_{k}}\right)^{1 / M}=g_{1}(y)<\infty .
\end{aligned}
$$

This means that $x \in N^{t}(p)$. Consequently, $T$ is surjective and is paranorm preserving. Hence, $T$ is linear bijection and this says us that the spaces $N^{t}(p)$ and $\ell(p)$ are linearly isomorphic. Therefore, the proof is completed.

We determine the basis for the paranormed space $N^{t}(p)$.

Theorem 4. Define the sequence $b^{(k)}(t)=\left\{b_{n}^{(k)}(t)\right\}_{n \in \mathbb{N}}$ of the elements of the space $N^{t}(p)$ for every fixed $k \in \mathbb{N}$ by

$$
b_{n}^{(k)}(t)= \begin{cases}(-1)^{n-k} D_{n-k} T_{k}, & 0 \leq k \leq n, \\ 0, & k>n .\end{cases}
$$

Then, the sequence $\left\{b^{(k)}(t)\right\}_{k \in \mathbb{N}}$ is a basis for the space $N^{t}(p)$ and any $x \in N^{t}(p)$ has a unique representation of the form

$$
x=\sum_{k} \lambda_{k}(t) b^{(k)}(t),
$$

where $\lambda_{k}(t)=\left(N^{t} x\right)_{k}$ for all $k \in \mathbb{N}$ and $0<p_{k} \leq H<\infty$.

Proof. It is clear that $\left\{b^{(k)}(t)\right\} \subset N^{t}(p)$, since

$$
N^{t} b^{(k)}(t)=e^{(k)} \in \ell(p) \quad \forall k \in \mathbb{N}
$$

where $e^{(k)}$ is the sequence whose only nonzero term is a 1 in the $k$ th place for each $k \in \mathbb{N}$ and $0<p_{k} \leq H<\infty$.

Let $x \in N^{t}(p)$ be given. For every nonnegative integer $m$, we put

$$
x^{[m]}=\sum_{k=0}^{m} \lambda_{k}(t) b^{(k)}(t) .
$$

Then, we obtain by applying $N^{t}$ to (25) with (24) that

$$
\begin{gathered}
N^{t} x^{[m]}=\sum_{k=0}^{m} \lambda_{k}(t) N^{t} b^{(k)}(t)=\sum_{k=0}^{m}\left(N^{t} x\right)_{k} e^{(k)}, \\
\left\{N^{t}\left(x-x^{[m]}\right)\right\}_{i}= \begin{cases}0, & 0 \leq i \leq m, \\
\left(N^{t} x\right)_{i}, & i>m,\end{cases}
\end{gathered}
$$


where $i, m \in \mathbb{N}$. Given $\epsilon>0$, then there is an integer $m_{0}$ such that

$$
\left[\sum_{i=m+1}^{\infty}\left|\left(N^{t} x\right)_{i}\right|^{p_{k}}\right]^{1 / M}<\epsilon
$$

for all $(m+1) \geq m_{0}$. Hence,

$$
\begin{aligned}
g\left[N^{t}\left(x-x^{[m]}\right)\right] & =\left[\sum_{i=m+1}^{\infty}\left|\left(N^{t} x\right)_{i}\right|^{p_{k}}\right]^{1 / M} \\
& \leq\left[\sum_{i=m_{0}}^{\infty}\left|\left(N^{t} x\right)_{i}\right|^{p_{k}}\right]^{1 / M}<\epsilon
\end{aligned}
$$

for all $(m+1) \geq m_{0}$ which proves that $x \in N^{t}(p)$ is represented as in (23).

Let us show the uniqueness of the representation for $x \in$ $N^{t}(p)$ given by (23). Suppose, on the contrary, that there exists a representation $x=\sum_{k} \mu_{k}(t) b^{(k)}(t)$. Since the linear transformation $T$, from $N^{t}(p)$ to $\ell(p)$, used in the proof of Theorem 3 is continuous, we have at this stage that

$$
\left(N^{t} x\right)_{n}=\sum_{k} \mu_{k}(t)\left\{N^{t} b^{(k)}(t)\right\}_{n}=\sum_{k} \mu_{k}(t) e_{n}^{(k)}=\mu_{n}(t)
$$

for all $n \in \mathbb{N}$ which contradicts the fact that $\left(N^{t} x\right)_{n}=\lambda_{n}(t)$ for all $n \in \mathbb{N}$. Hence, the representation (23) of $x \in N^{t}(p)$ is unique. This completes the proof.

\section{The Alpha-, Beta-, and Gamma-Duals of the Space $N^{t}(p)$}

In this section, we determine the alpha-, beta-, and gammaduals of the space $N^{t}(p)$. We will quote some lemmas which are needed in proving our theorems.

Lemma 5 (see [11], Theorem 5.1.0). The following statements hold.

(i) Let $1<p_{k} \leq H<\infty$ for every $k \in \mathbb{N}$. Then, $A=$ $\left(a_{n k}\right) \in\left(\ell(p): \ell_{1}\right)$ if and only if there exists an integer $B>1$ such that

$$
\sup _{N \in \mathscr{F}} \sum_{k}\left|\sum_{n \in N} a_{n k} B^{-1}\right|^{p_{k}^{\prime}}<\infty .
$$

(ii) Let $0<p_{k} \leq 1$ for every $k \in \mathbb{N}$. Then, $A=\left(a_{n k}\right) \in$ $\left(\ell(p): \ell_{1}\right)$ if and only if

$$
\left.\sup _{N \in \mathscr{F}}\left|\sup _{k \in \mathbb{N}}\right| \sum_{n \in N} a_{n k}\right|^{p_{k}}<\infty .
$$

Lemma 6 (see [12], Theorem 1). The following statements hold.

(i) Let $1<p_{k} \leq H<\infty$ for every $k \in \mathbb{N}$. Then, $A=$ $\left(a_{n k}\right) \in\left(\ell(p): \ell_{\infty}\right)$ if and only if there exists an integer $B>1$ such that

$$
\sup _{n \in \mathbb{N}} \sum_{k}\left|a_{n k} B^{-1}\right|^{p_{k}^{\prime}}<\infty
$$

(ii) Let $0<p_{k} \leq 1$ for every $k \in \mathbb{N}$. Then, $A=\left(a_{n k}\right) \in$ $\left(\ell(p): \ell_{\infty}\right)$ if and only if

$$
\sup _{n, k \in \mathbb{N}}\left|a_{n k}\right|^{p_{k}}<\infty .
$$

Lemma 7 (see [12], Theorem 1). Let $0<p_{k} \leq H<\infty$ for every $k \in \mathbb{N}$. Then, $A=\left(a_{n k}\right) \in(\ell(p): c)$ if and only if (32), (33) hold and there is $\beta_{k} \in \mathbb{C}$ such that $a_{n k} \rightarrow \beta_{k}$ for each $k \in \mathbb{N}$.

Theorem 8. Let $1<p_{k} \leq H<\infty$ for every $k \in \mathbb{N}$. Define the sets $D_{1}(p), D_{2}(p)$, and $D_{3}(p)$ as follows:

$$
\begin{aligned}
& D_{1}(p):=\left\{a=\left(a_{k}\right) \in \omega\right. \\
& \quad\left.\quad \sup _{N \in \mathscr{F}} \sum_{k}\left|\sum_{n \in N}(-1)^{n-k} a_{n} D_{n-k} T_{k} B^{-1}\right|^{p_{k}^{\prime}}<\infty\right\}, \\
& D_{2}(p):=\left\{a=\left(a_{k}\right) \in \omega: \sup _{n \in \mathbb{N}} \sum_{k}\left|\sum_{i=k}^{n}(-1)^{i-k} a_{i} D_{i-k} T_{k} B^{-1}\right|^{p_{k}^{\prime}}\right. \\
&\left.<\infty,\left\{\left(a_{n} T_{n} B^{-1}\right)^{p_{k}^{\prime}}\right\} \in \ell_{\infty}\right\}, \\
& D_{3}(p)=c s .
\end{aligned}
$$

Then, the following statements hold:

(i) $\left\{N^{t}(p)\right\}^{\alpha}=D_{1}(p)$;

(ii) $\left\{N^{t}(p)\right\}^{\gamma}=D_{2}(p)$;

(iii) $\left\{N^{t}(p)\right\}^{\beta}=D_{2}(p) \cap D_{3}(p)$.

Proof. (i) Let us take $a=\left(a_{k}\right) \in \omega$. We easily derive with (20) that

$$
a_{n} x_{n}=\sum_{k=0}^{n}(-1)^{n-k} a_{n} D_{n-k} T_{k} y_{k}=(C y)_{n} \quad \forall n \in \mathbb{N},
$$


where $C=\left(c_{n k}\right)$ is defined by

$$
c_{n k}= \begin{cases}(-1)^{n-k} a_{n} D_{n-k} T_{k}, & 0 \leq k \leq n, \\ 0, & k>n\end{cases}
$$

for all $k, n \in \mathbb{N}$. Thus, we observe by combining (35) with Part (i) of Lemma 5 that $a x=\left(a_{n} x_{n}\right) \in \ell_{1}$ whenever $x=\left(x_{k}\right) \in$ $N^{t}(p)$ if and only if $C y \in \ell_{1}$ whenever $y=\left(y_{k}\right) \in \ell(p)$. This gives the result that $\left\{N^{t}(p)\right\}^{\alpha}=D_{1}(p)$.

(ii) Consider the equality

$$
\begin{aligned}
\sum_{k=0}^{n} a_{k} x_{k} & =\sum_{k=0}^{n-1} \sum_{i=k}^{n}(-1)^{i-k} a_{i} D_{i-k} T_{k} y_{k}+a_{n} T_{n} y_{n} \\
& =(E y)_{n} \quad \forall n \in \mathbb{N},
\end{aligned}
$$

where $E=\left(e_{n k}\right)$ is defined by

$$
e_{n k}= \begin{cases}\sum_{i=k}^{n}(-1)^{i-k} a_{i} D_{i-k} T_{k}, & 0 \leq k \leq n-1, \\ a_{n} T_{n}, & k=n, \\ 0, & k>n\end{cases}
$$

for all $k, n \in \mathbb{N}$. Thus, we deduce from Part (i) of Lemma 6 with (37) that $a x=\left(a_{n} x_{n}\right) \in b s$ whenever $x=\left(x_{k}\right) \in N^{t}(p)$ if and only if $E y \in \ell_{\infty}$ whenever $y=\left(y_{k}\right) \in \ell(p)$. Therefore, we obtain from Part (i) of Lemma 6 that $\left\{N^{t}(p)\right\}^{\gamma}=D_{2}(p)$.

(iii) We see from Lemma 7 that $a x=\left(a_{n} x_{n}\right) \in c s$ whenever $x=\left(x_{k}\right) \in N^{t}(p)$ if and only if $E y \in c$ whenever $y=\left(y_{k}\right) \in \ell(p)$. Therefore, we derive from Lemma 7 that $\left\{N^{t}(p)\right\}^{\beta}=D_{2}(p) \cap D_{3}(p)$.

Therefore, the proof is completed.

Theorem 9. Let $0<p_{k} \leq 1$ for every $k \in \mathbb{N}$. Define the sets $D_{4}(p)$ and $D_{5}(p)$ by

$$
\begin{aligned}
D_{4}(p):= & \left\{a=\left(a_{k}\right) \in \omega\right. \\
& \left.\quad \sup _{N \in \mathscr{F}} \sup _{k \in \mathbb{N}}\left|\sum_{n \in N}(-1)^{n-k} a_{n} D_{n-k} T_{k}\right|^{p_{k}}<\infty\right\}, \\
D_{5}(p):=\left\{a=\left(a_{k}\right) \in \omega:\left.\sup _{n, k \in \mathbb{N}}\left|\sum_{i=k}^{n}(-1)^{i-k} a_{i} D_{i-k} T_{k}\right|\right|^{p_{k}}\right. & \\
& \left.<\infty,\left\{\left(a_{n} T_{n}\right)^{p_{k}}\right\} \in \ell_{\infty}\right\} .
\end{aligned}
$$

Then, the following statements hold:

(i) $\left\{N^{t}(p)\right\}^{\alpha}=D_{4}(p)$;

(ii) $\left\{N^{t}(p)\right\}^{\gamma}=D_{5}(p)$;

(iii) $\left\{N^{t}(p)\right\}^{\beta}=D_{3}(p) \cap D_{5}(p)$.
Proof. This is easily obtained by proceeding as in the proof of Theorem 8 by using Lemma 7 and the second parts of Lemmas 5 and 6 instead of the first parts. So, we omit the detail.

\section{Some Matrix Transformations Related to the Sequence Space $N^{t}(p)$}

In the present section, we characterize the matrix transformations from the space $N^{t}(p)$ into any given sequence space $\mu$ and from a given sequence space $\mu$ into the space $N^{t}(p)$. Since $\mu_{A} \cong \mu$ for any triangle $A$ and any sequence space $\mu$, it is trivial that the equivalence " $x \in \mu_{A}$ if and only if $y=A x \in \mu$ " holds.

Now, we can give the following theorem.

Theorem 10. Suppose that the elements of the infinite matrices $A=\left(a_{n k}\right)$ and $F=\left(f_{n k}\right)$ are connected with the relation

$$
f_{n k}:=\sum_{j=k}^{\infty}(-1)^{j-k} D_{j-k} T_{k} a_{n j}
$$

for all $k, n \in \mathbb{N}$ and $\mu$ is any given sequence space. Then, $A \in$ $\left(N^{t}(p): \mu\right)$ if and only if $A_{n} \in\left\{N^{t}(p)\right\}^{\beta}$ for all $n \in \mathbb{N}$ and $F \in(\ell(p): \mu)$.

Proof. Let $\mu$ be any given sequence space. Suppose that (40) holds between the elements of the matrices $A=\left(a_{n k}\right)$ and $F=\left(f_{n k}\right)$, and take into account that the spaces $N^{t}(p)$ and $\ell(p)$ are linearly isomorphic.

Let $A \in\left(N^{t}(p): \mu\right)$ and take any $y \in \ell(p)$. Then

$$
\left(F N^{t}\right)_{n k}=\sum_{j=k}^{\infty} f_{n j} a_{j k}^{t}=\sum_{j=k}^{\infty} \sum_{i=j}^{\infty}(-1)^{i-j} D_{i-j} a_{n i} T_{j} \frac{t_{j-k}}{T_{j}}=a_{n k} .
$$

That is, $F N^{t}$ exists and $A_{n} \in\left\{N^{t}(p)\right\}^{\beta}$ which yields that $F_{n} \in$ $\ell_{1}$ for each $n \in \mathbb{N}$. Hence, $F y$ exists and thus

$$
\begin{aligned}
\sum_{k} f_{n k} y_{k}= & \sum_{k} \sum_{i=k}^{\infty}(-1)^{i-k} D_{i-k} a_{n i} T_{k} \\
& \times\left(\frac{1}{T_{k}} \sum_{j=0}^{k} t_{k-j} x_{j}\right)=\sum_{k} a_{n k} x_{k}
\end{aligned}
$$

for all $n \in \mathbb{N}$. So, we have that $F y=A x$, which leads us to the consequence $F \in(\ell(p): \mu)$.

Conversely, let $A_{n} \in\left\{N^{t}(p)\right\}^{\beta}$ for each $n \in \mathbb{N}$ and $F \in(\ell(p): \mu)$, and take $x=\left(x_{k}\right) \in N^{t}(p)$. Then, $A x$ exists. Therefore, we obtain from the equality

$$
\sum_{k} a_{n k} x_{k}=\sum_{k} a_{n k}\left[\sum_{i=0}^{k}(-1)^{k-i} D_{k-i} T_{i} y_{i}\right]=\sum_{k} f_{n k} y_{k} \quad \forall n \in \mathbb{N}
$$

that $A x=F y$ and this shows that $A \in\left(N^{t}(p): \mu\right)$. This completes the proof. 
By changing the roles of the spaces $N^{t}(p)$ with $\mu$ in Theorem 10, we have the following.

Theorem 11. Suppose that $\mu$ is any given sequence space and the elements of the infinite matrices $A=\left(a_{n k}\right)$ and $G=\left(g_{n k}\right)$ are connected with the relation $g_{n k}=\sum_{j=0}^{n}\left(t_{n-j} / T_{n}\right) a_{j k}$ for all $k, n \in \mathbb{N}$. Then, $A \in\left(\mu: N^{t}(p)\right)$ if and only if $G \in(\mu: \ell(p))$.

Proof. Let $x=\left(x_{k}\right) \in \mu$ and consider the following equality:

$$
\sum_{j=0}^{n} \frac{t_{n-j}}{T_{n}} \sum_{k=0}^{m} a_{j k} x_{k}=\sum_{k=0}^{m} g_{n k} x_{k} \quad \forall n \in \mathbb{N} .
$$

Then, by letting $m \rightarrow \infty$ in (44), we have $\left\{N^{t}(A x)\right\}_{n}=(G x)_{n}$ for all $n \in \mathbb{N}$. Since $A x \in N^{t}(p), N^{t}(A x)=G x \in \ell(p)$. This completes the proof.

\section{The Rotundity of the Space $N^{t}(p)$}

In functional analysis, the rotundity of Banach spaces is one of the most important geometric properties. For details, the reader may refer to [13-15]. In this section, we give the necessary and sufficient condition in order to the space $N^{t}(p)$ be rotund and present some results related to this concept.

Definition 12. Let $S(X)$ be the unit sphere of a Banach space $X$. Then, a point $x \in S(X)$ is called an extreme point if $2 x=$ $y+z$ implies $y=z$ for every $y, z \in S(X)$. A Banach space $X$ is said to be rotund (strictly convex) if every point of $S(X)$ is an extreme point.

Definition 13. A Banach space $X$ is said to have KadecKlee property (or property $(H)$ ) if every weakly convergent sequence on the unit sphere is convergent in norm.

Definition 14. A Banach space $X$ is said to have

(i) the Opial property if every sequence $\left(x_{n}\right)$ weakly convergent to $x_{0} \in X$ satisfies

$$
\liminf _{n \rightarrow \infty}\left\|x_{n}-x_{0}\right\|<\liminf _{n \rightarrow \infty}\left\|x_{n}+x\right\|
$$

for every $x \in X$ with $x \neq x_{0}$;

(ii) the uniform Opial property if for each $\epsilon>0$, there exists an $r>0$ such that

$$
1+r \leq \liminf _{n \rightarrow \infty}\left\|x_{n}+x\right\|
$$

for each $x \in X$ with $\|x\| \geq \epsilon$ and each sequence $\left(x_{n}\right)$ in $X$ such that $x_{n} \stackrel{w}{\rightarrow} 0$ and $\lim \inf _{n \rightarrow \infty}\left\|x_{n}\right\| \geq 1$.

Definition 15. Let $X$ be a real vector space. A functional $\sigma$ : $X \rightarrow[0, \infty)$ is called a modular if

(i) $\sigma(x)=0$ if and only if $x=\theta$;

(ii) $\sigma(\alpha x)=\sigma(x)$ for all scalars $\alpha$ with $|\alpha|=1$;

(iii) $\sigma(\alpha x+\beta y) \leq \sigma(x)+\sigma(y)$ for all $x, y \in X$ and $\alpha, \beta \geq 0$ with $\alpha+\beta=1$; (iv) the modular $\sigma$ is called convex if $\sigma(\alpha x+\beta y) \leq \alpha \sigma(x)+$ $\beta \sigma(y)$ for all $x, y \in X$ and $\alpha, \beta>0$ with $\alpha+\beta=1$.

A modular $\sigma$ on $X$ is called

(a) right continuous if $\lim _{\alpha \rightarrow 1^{+}} \sigma(\alpha x)=\sigma(x)$ for all $x \in X_{\sigma}$;

(b) left continuous if $\lim _{\alpha \rightarrow 1^{-}} \sigma(\alpha x)=\sigma(x)$ for all $x \in X_{\sigma}$;

(c) continuous if it is both right and left continuous, where

$$
X_{\sigma}=\left\{x \in X: \lim _{\alpha \rightarrow 0^{+}} \sigma(\alpha x)=0\right\} .
$$

We define $\sigma_{p}$ on $N^{t}(p)$ by $\sigma_{p}(x)=$ $\sum_{k}\left|\left(1 / T_{k}\right) \sum_{j=0}^{k} t_{k-j} x_{j}\right|^{p_{k}}$. If $p_{k} \geq 1$ for all $k \in \mathbb{N}_{1}=\{1,2, \ldots\}$, by the convexity of the function $t \mapsto|t|^{p_{k}}$ for each $k \in \mathbb{N}, \sigma_{p}$ is a convex modular on $N^{t}(p)$. We consider $N^{t}(p)$ equipped with Luxemburg norm given by

$$
\|x\|=\inf \left\{\alpha>0: \sigma_{p}\left(\frac{x}{\alpha}\right) \leq 1\right\} .
$$

$N^{t}(p)$ is a Banach space with this norm. This can be shown by the similar way used in the proof of Theorem 7 in [16].

We establish some basic properties for the modular $\sigma_{p}$.

Proposition 16. The modular $\sigma_{p}$ on $N^{t}(p)$ satisfies the following properties with $p_{k} \geq 1$ for all $k \in \mathbb{N}$.

(i) If $0<\alpha \leq 1$, then $\alpha^{M} \sigma_{p}(x / \alpha) \leq \sigma_{p}(x)$ and $\sigma_{p}(\alpha x) \leq$ $\alpha \sigma_{p}(x)$.

(ii) If $\alpha \geq 1$, then $\sigma_{p}(x) \leq \alpha^{M} \sigma_{p}(x / \alpha)$.

(iii) If $\alpha \geq 1$, then $\alpha \sigma_{p}(x / \alpha) \leq \sigma_{p}(x)$.

(iv) The modular $\sigma_{p}$ is continuous.

Proof. (i) Let $0<\alpha \leq 1$. Then $\alpha^{M} / \alpha^{p_{k}} \leq 1$ for all $p_{k} \geq 1$. So, we have

$$
\begin{aligned}
\alpha^{M} \sigma_{p}\left(\frac{x}{\alpha}\right) & =\sum_{k} \frac{\alpha^{M}}{\alpha_{k}}\left|\frac{1}{T_{k}} \sum_{j=0}^{k} t_{k-j} x_{j}\right|^{p_{k}} \\
& \leq \sum_{k}\left|\frac{1}{T_{k}} \sum_{j=0}^{k} t_{k-j} x_{j}\right|^{p_{k}}=\sigma_{p}(x), \\
\sigma_{p}(\alpha x) & =\sum_{k} \alpha^{p_{k}}\left|\frac{1}{T_{k}} \sum_{j=0}^{k} t_{k-j} x_{j}\right|^{p_{k}} \\
& \leq \alpha \sum_{k}\left|\frac{1}{T_{k}} \sum_{j=0}^{k} t_{k-j} x_{j}\right|^{p_{k}}=\alpha \sigma_{p}(x) .
\end{aligned}
$$

(ii) Let $\alpha \geq 1$. Then $1 \leq \alpha^{M} / \alpha^{p_{k}}$ for all $p_{k} \geq 1$. So, we have

$$
\sigma_{p}(x) \leq \frac{\alpha^{M}}{\alpha^{p_{k}}} \sigma_{p}(x)=\alpha^{M} \sigma_{p}\left(\frac{x}{\alpha}\right) .
$$


(iii) Let $\alpha \geq 1$. Then $\alpha / \alpha^{p_{k}} \leq 1$ for all $p_{k} \geq 1$. Therefore, one can easily see that

$$
\begin{aligned}
\alpha \sigma_{p}\left(\frac{x}{\alpha}\right) & =\sum_{k} \frac{\alpha}{\alpha^{p_{k}}}\left|\frac{1}{T_{k}} \sum_{j=0}^{k} t_{k-j} x_{j}\right|^{p_{k}} \\
& \leq \sum_{k}\left|\frac{1}{T_{k}} \sum_{j=0}^{k} t_{k-j} x_{j}\right|^{p_{k}}=\sigma_{p}(x) .
\end{aligned}
$$

(iv) If $\alpha>1$, then we have

$$
\begin{aligned}
\sum_{k} \alpha\left|\frac{1}{T_{k}} \sum_{j=0}^{k} t_{k-j} x_{j}\right|^{p_{k}} & \leq \sum_{k} \alpha^{p_{k}}\left|\frac{1}{T_{k}} \sum_{j=0}^{k} t_{k-j} x_{j}\right|^{p_{k}} \\
& \leq \sum_{k} \alpha^{M}\left|\frac{1}{T_{k}} \sum_{j=0}^{k} t_{k-j} x_{j}\right|^{p_{k}} ;
\end{aligned}
$$

that is,

$$
\alpha \sigma_{p}(x) \leq \sigma_{p}(\alpha x) \leq \alpha^{M} \sigma_{p}(x) .
$$

By passing to limit as $\alpha \rightarrow 1^{+}$in (53), we have $\sigma_{p}(\alpha x) \rightarrow$ $\sigma_{p}(x)$. Hence, $\sigma_{p}$ is right continuous.

If $0<\alpha<1$, we have

$$
\begin{aligned}
\sum_{k} \alpha^{M}\left|\frac{1}{T_{k}} \sum_{j=0}^{k} t_{k-j} x_{j}\right|^{p_{k}} & \leq \sum_{k} \alpha^{p_{k}}\left|\frac{1}{T_{k}} \sum_{j=0}^{k} t_{k-j} x_{j}\right|^{p_{k}} \\
& \leq \sum_{k} \alpha\left|\frac{1}{T_{k}} \sum_{j=0}^{k} t_{k-j} x_{j}\right|^{p_{k}} ;
\end{aligned}
$$

that is,

$$
\alpha^{M} \sigma_{p}(x) \leq \sigma_{p}(\alpha x) \leq \alpha \sigma_{p}(x) .
$$

By letting $\alpha \rightarrow 1^{-}$in (55), we have $\sigma_{p}(\alpha x) \rightarrow \sigma_{p}(x)$. Hence, $\sigma_{p}$ is left continuous. Since $\sigma_{p}$ is both right and left continuous, it is continuous.

Now, we give some relationships between the modular $\sigma_{p}$ and the Luxemburg norm on $N^{t}(p)$.

Proposition 17. For any $x \in N^{t}(p)$, the following statements hold.

(i) If $\|x\|<1$, then $\sigma_{p}(x) \leq\|x\|$.

(ii) If $\|x\|>1$, then $\sigma_{p}(x) \geq\|x\|$.

(iii) $\|x\|=1$ if and only if $\sigma_{p}(x)=1$.

(iv) $\|x\|<1$ if and only if $\sigma_{p}(x)<1$.

(v) $\|x\|>1$ if and only if $\sigma_{p}(x)>1$.

(vi) If $0<\alpha<1$ and $\|x\|>\alpha$, then $\sigma_{p}(x)>\alpha^{M}$.

(vii) If $\alpha \geq 1$ and $\|x\|<\alpha$, then $\sigma_{p}(x)<\alpha^{M}$.
Proof. Let $x \in N^{t}(p)$.

(i) Let $\epsilon>0$ such that $0<\epsilon<1-\|x\|$. By the definition of $\|\cdot\|$ in (48), there exists an $\alpha>0$ such that $\|x\|+\epsilon>\alpha$ and $\sigma_{p}(x / \alpha) \leq 1$. So, we have

$$
\begin{aligned}
\sigma_{p}(x) & \leq \sum_{k}\left(\frac{\|x\|+\epsilon}{\alpha}\right)^{p_{k}}\left|\frac{1}{T_{k}} \sum_{j=0}^{k} t_{k-j} x_{j}\right|^{p_{k}} \\
& \leq(\|x\|+\epsilon) \sigma_{p}\left(\frac{x}{\alpha}\right) \leq\|x\|+\epsilon .
\end{aligned}
$$

Since $\epsilon$ is arbitrary, we have $\sigma_{p}(x) \leq\|x\|$ from (56).

(ii) If we choose $\epsilon>0$ such that $0<\epsilon<1-1 /\|x\|$, then $1<(1-\epsilon)\|x\|<\|x\|$. By the definition of $\|\cdot\|$ in (48) and Part (iii) of Proposition 16, we have

$$
1<\sigma_{p}\left[\frac{x}{(1-\epsilon)\|x\|}\right] \leq \frac{1}{(1-\epsilon)\|x\|} \sigma_{p}(x) .
$$

So, $(1-\epsilon)\|x\|<\|x\|$ for all $\epsilon \in(0,1-(1 /\|x\|))$. This implies that $\|x\|<\sigma_{p}(x)$.

(iii) Since $\sigma_{p}$ is continuous, by Theorem 1.4 of [15] we directly have (iii).

(iv) This follows from Parts (i) and (iii).

(v) This follows from Parts (ii) and (iii).

(vi) This follows from Part (ii) and Part (i) of Proposition 16.

(vii) This follows from Part (i) and Part (ii) of Proposition 16.

Theorem 18. The space $N^{t}(p)$ is rotund if and only if $p_{k}>1$ for all $k \in \mathbb{N}$.

Proof. Let $N^{t}(p)$ be rotund and choose $k \in \mathbb{N}$ such that $p_{k}=1$ for all $k<3$. Consider the following sequences given by

$$
\begin{gathered}
x=\left(1,-D_{1}, D_{2},-D_{3}, D_{4}, \ldots\right), \\
y=\left(0, T_{1},-T_{1} D_{1}, T_{1} D_{2},-T_{1} D_{3}, \ldots\right) .
\end{gathered}
$$

Then, obviously $x \neq y$ and

$$
\sigma_{p}(x)=\sigma_{p}(y)=\sigma_{p}\left(\frac{x+y}{2}\right)=1 .
$$

By Part (iii) of Proposition 17, $x, y,(x+y) / 2 \in S\left[N^{t}(p)\right]$ which leads us to the contradiction that the sequence space $N^{t}(p)$ is not rotund. Hence, $p_{k}>1$ for all $k \in \mathbb{N}$.

Conversely, let $x \in S\left[N^{t}(p)\right]$ and $v, z \in S\left[N^{t}(p)\right]$ with $x=$ $(v+z) / 2$. By convexity of $\sigma_{p}$ and Part (iii) of Proposition 17, we have

$$
1=\sigma_{p}(x) \leq \frac{\sigma_{p}(v)+\sigma_{p}(z)}{2}=1,
$$


which gives that

$$
\sigma_{p}(x)=\frac{\sigma_{p}(v)+\sigma_{p}(z)}{2} .
$$

Also, since $x=(v+z) / 2$ and from (61), we obtain that

$$
\begin{aligned}
& \sum_{k}\left|\frac{1}{T_{k}} \sum_{j=0}^{k} t_{k-j} \frac{\left(v_{j}+z_{j}\right)}{2}\right|^{p_{k}} \\
& =\frac{1}{2}\left(\sum_{k}\left|\frac{1}{T_{k}} \sum_{j=0}^{k} t_{k-j} v_{j}\right|^{p_{k}}+\sum_{k}\left|\frac{1}{T_{k}} \sum_{j=0}^{k} t_{k-j} z_{j}\right|^{p_{k}}\right) .
\end{aligned}
$$

This implies that

$$
\left|\frac{v_{j}+z_{j}}{2}\right|^{p_{k}}=\frac{\left|v_{j}\right|^{p_{k}}+\left|z_{j}\right|^{p_{k}}}{2}
$$

for all $k \in \mathbb{N}$. Since the function $t \rightarrow|t|^{p_{k}}$ is strictly convex for all $k \in \mathbb{N}$, it follows by (63) that $v_{k}=z_{k}$ for all $k \in \mathbb{N}$. Hence, $v=z$. That is, $N^{t}(p)$ is rotund.

Theorem 19. Let $\left(x_{n}\right)$ be a sequence in $N^{t}(p)$. Then, the following statements hold:

(i) $\lim _{n \rightarrow \infty}\left\|x_{n}\right\|=1$ implies $\lim _{n \rightarrow \infty} \sigma_{p}\left(x_{n}\right)=1$;

(ii) $\lim _{n \rightarrow \infty} \sigma_{p}\left(x_{n}\right)=0$ implies $\lim _{n \rightarrow \infty}\left\|x_{n}\right\|=0$.

Proof. The proof is similar to that of Theorem 10 in [16].

Theorem 20. Let $x \in N^{t}(p)$ and $\left(x^{(n)}\right) \subset N^{t}(p)$. If $\sigma_{p}\left(x^{(n)}\right) \rightarrow \sigma_{p}(x)$ as $n \rightarrow \infty$ and $x_{k}^{(n)} \rightarrow x_{k}$ as $n \rightarrow \infty$ for all $k \in \mathbb{N}$, then $x^{(n)} \rightarrow x$ as $n \rightarrow \infty$.

Proof. Let $\epsilon>0$ be given. Since $x \in N^{t}(p)$ and $\left(x^{(n)}\right) \subset N^{t}(p)$, $\sigma_{p}\left(x^{(n)}-x\right)=\sum_{k}\left|\left\{N^{t}\left(x^{(n)}-x\right)\right\}_{k}\right|^{p_{k}}<\infty$. So, there exists an $k_{0} \in \mathbb{N}$ such that

$$
\sum_{k=k_{0}+1}^{\infty}\left|\left\{N^{t}\left(x^{(n)}-x\right)\right\}_{k}\right|^{p_{k}}<\frac{\epsilon}{2}
$$

Also, since $x_{k}^{(n)} \rightarrow x_{k}$ as $n \rightarrow \infty$, we have

$$
\sum_{k=1}^{k_{0}}\left|\left\{N^{t}\left(x^{(n)}-x\right)\right\}_{k}\right|^{p_{k}}<\frac{\epsilon}{2} .
$$

Therefore, we obtain from (64) and (65) that $\sigma_{p}\left(x^{(n)}-x\right)<\epsilon$. This means that $\sigma_{p}\left(x^{(n)}-x\right) \rightarrow 0$ as $n \rightarrow \infty$. This result implies $\left\|x^{(n)}-x\right\| \rightarrow 0$ as $n \rightarrow \infty$ from Part (ii) of Theorem 19. Hence, $x_{n} \rightarrow x$ as $n \rightarrow \infty$.

Theorem 21. The sequence space $N^{t}(p)$ has the Kadec-Klee property.

Proof. Let $x \in S\left[N^{t}(p)\right]$ and $\left(x^{(n)}\right) \subset N^{t}(p)$ such that $\left\|x^{(n)}\right\| \rightarrow 1$ and $x^{(n)} \stackrel{w}{\rightarrow} x$ are given. By Part (i) of Theorem 19, we have $\sigma_{p}\left(x^{(n)}\right) \rightarrow 1$ as $n \rightarrow \infty$. Also, $x \in S\left[N^{t}(p)\right]$ implies $\|x\|=1$. By Part (iii) of Proposition 17, we obtain $\sigma_{p}(x)=1$. Therefore, we have $\sigma_{p}\left(x^{(n)}\right) \rightarrow \sigma_{p}(x)$ as $n \rightarrow \infty$.

Since $x^{(n)} \stackrel{w}{\rightarrow} x$ and $q_{k}: N^{t}(p) \rightarrow \mathbb{R}($ or $\mathbb{C}$ ) defined by $q_{k}(x)=x_{k}$ is continuous, $x_{k}^{(n)} \rightarrow x_{k}$ as $n \rightarrow \infty$. Therefore, $x^{(n)} \rightarrow x$ as $n \rightarrow \infty$. This completes the proof.

Theorem 22. For any $1<p<\infty$, the space $X_{a(p)}$ has the uniform Opial property.

Proof. Since the proof can be given by the similar way used in proving Theorem 13 of Nergiz and Başar [16], we omit the detail.

\section{Conclusion}

Wang introduced the sequence space $X_{a(p)}$, in [10]. Although the domain of several triangle matrices in the classical sequence spaces $\ell_{p}, c_{0}, c$, and $\ell_{\infty}$ and in the Maddox spaces $\ell(p), c_{0}(p), c(p)$, and $\ell_{\infty}(p)$ was investigated by researchers, the domain of Nörlund mean neither in a normed sequence space nor in a paranormed sequence space was not studied and is still as an open problem. So, we have worked on the domain of Nörlund mean in the Maddox space $\ell(p)$. Additionally, we emphasize on some geometric properties of the new space $N^{t}(p)$. It is obvious that the matrix $N^{t}$ is not comparable with the matrices $E^{r}, A^{r}$, or $B(r, s)$. So, the present results are new.

It is clear that by depending on the choice of the sequence space $\mu$, the characterization of several classes of matrix transformations from the space $N^{t}(p)$ and into the space $N^{t}(p)$ can be obtained from Theorems 10 and 11, respectively. As a natural continuation of this paper, we will study the domain of the Nörlund mean in Maddox's spaces $\ell_{\infty}(p)$, $c(p)$, and $c_{0}(p)$.

\section{Conflict of Interests}

The authors declare that there is no conflict of interests regarding the publication of this paper.

\section{References}

[1] I. J. Maddox, "Spaces of strongly summable sequences," Quarterly Journal of Mathematics, vol. 18, no. 1, pp. 345-355, 1967.

[2] H. Nakano, "Modulared sequence spaces," Proceedings of the Japan Academy, vol. 27, no. 2, pp. 508-512, 1951.

[3] S. Simons, "The sequence spaces $\ell\left(p_{v}\right)$ and $m\left(p_{v}\right)$," Proceedings of the London Mathematical Society, vol. 15, no. 3, pp. 422-436, 1965.

[4] A. Peyerimhoff, Lectures on Summability, Lecture Notes in Mathematics, Springer, New York, NY, USA, 1969.

[5] F. M. Mears, "The inverse Nörlund mean," Annals of Mathematics, vol. 44, no. 3, pp. 401-409, 1943.

[6] B. Choudhary and S. K. Mishra, "On Köthe-Toeplitz duals of certain sequence spaces and thair matrix transformations," Indian Journal of Pure and Applied Mathematics, vol. 24, no. 59, pp. 291-301, 1993. 
[7] F. Başar and B. Altay, "Matrix mappings on the space bs(p) and its $\alpha$-, $\beta$ - and $\gamma$-duals," The Aligarh Bulletin of Mathematics, vol. 21, no. 1, pp. 79-91, 2002.

[8] F. Başar, "Infinite matrices and almost boundedness," Bollettino della Unione Matematica Italiana A, vol. 6, no. 7, pp. 395-402, 1992.

[9] B. Altay and F. Başar, "On the paranormed Riesz sequence space of non-absolute type," Southeast Asian Bulletin of Mathematics, vol. 26, pp. 701-715, 2002.

[10] C. S. Wang, “On Nörlund sequence space," Tamkang Journal of Mathematics, vol. 9, pp. 269-274, 1978.

[11] K. G. Grosse-Erdmann, "Matrix transformations between the sequence spaces of Maddox," Journal of Mathematical Analysis and Applications, vol. 180, no. 1, pp. 223-238, 1993.

[12] C. G. Lascarides and I. J. Maddox, "Matrix transformations between some classes of sequences," Proceedings of the Cambridge Philosophical Society, vol. 68, pp. 99-104, 1970.

[13] S. Chen, "Geometry of Orlicz spaces," Dissertationes Mathematicae, vol. 356, pp. 1-224, 1996.

[14] J. Diestel, Geomety of Banach Spaces-Selected Topics, Springer, Berlin, Germany, 1984.

[15] L. Maligranda, Orlicz Spaces and Interpolation, Institute of Mathematics Polish Academy of Sciences, Poznan, Poland, 1985.

[16] H. Nergiz and F. Başar, "Some geometric properties of the domain of the double sequential band matrix $B(\widetilde{r}, \widetilde{s})$ in the sequence space $\ell(p)$," Abstract and Applied Analysis, vol. 2013, Article ID 421031, 7 pages, 2013. 


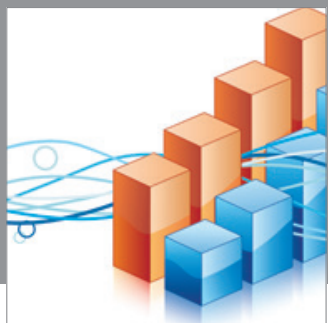

Advances in

Operations Research

mansans

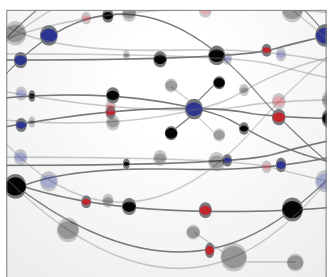

The Scientific World Journal
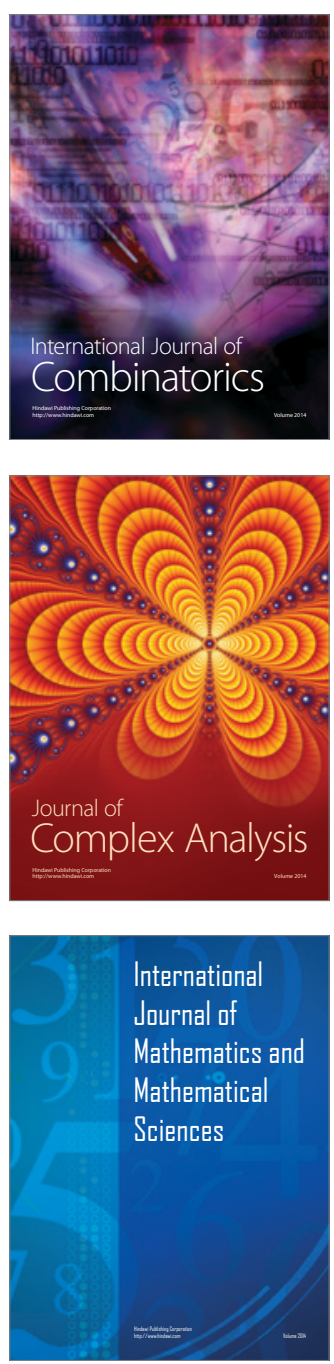
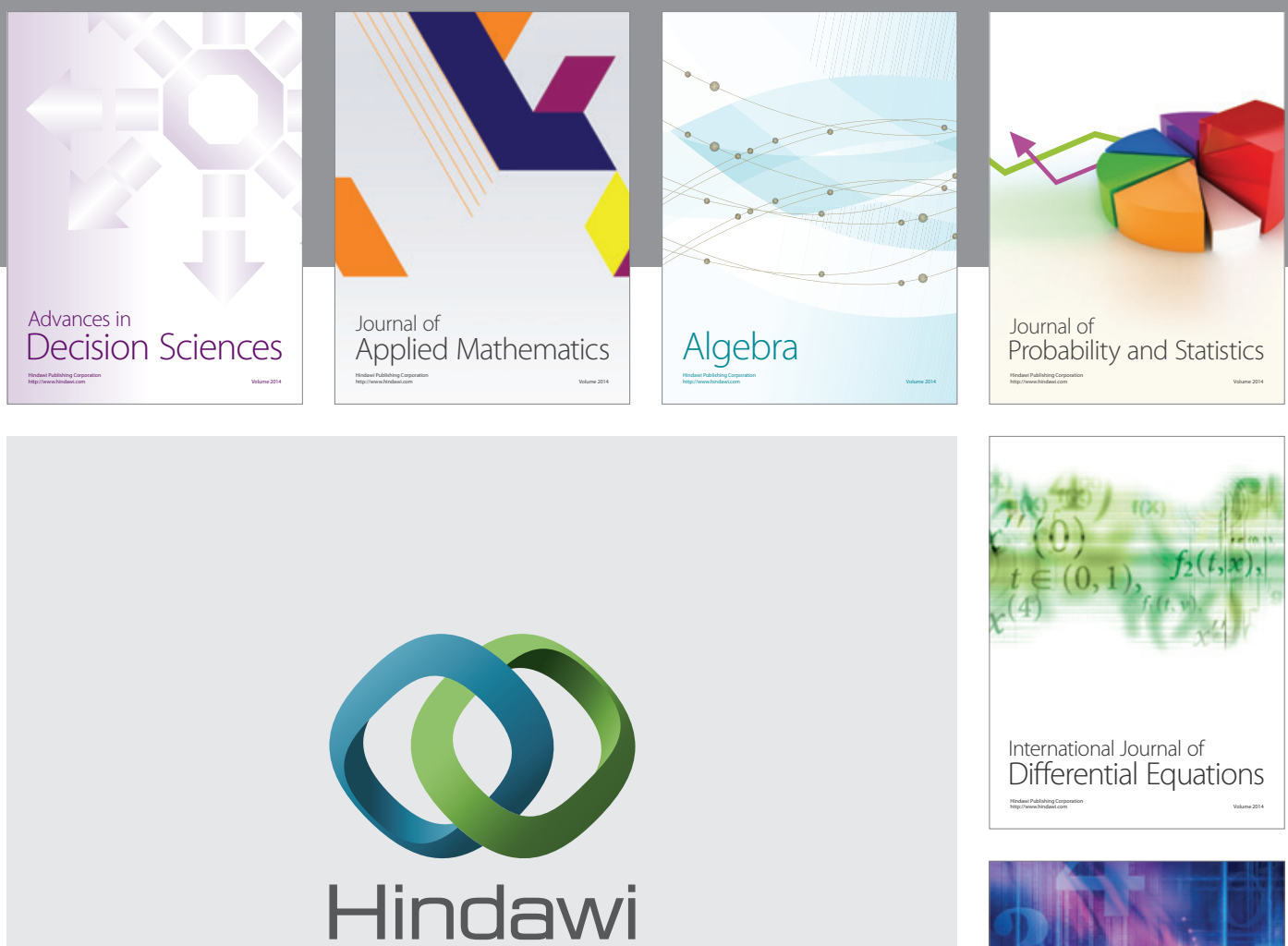

Submit your manuscripts at http://www.hindawi.com
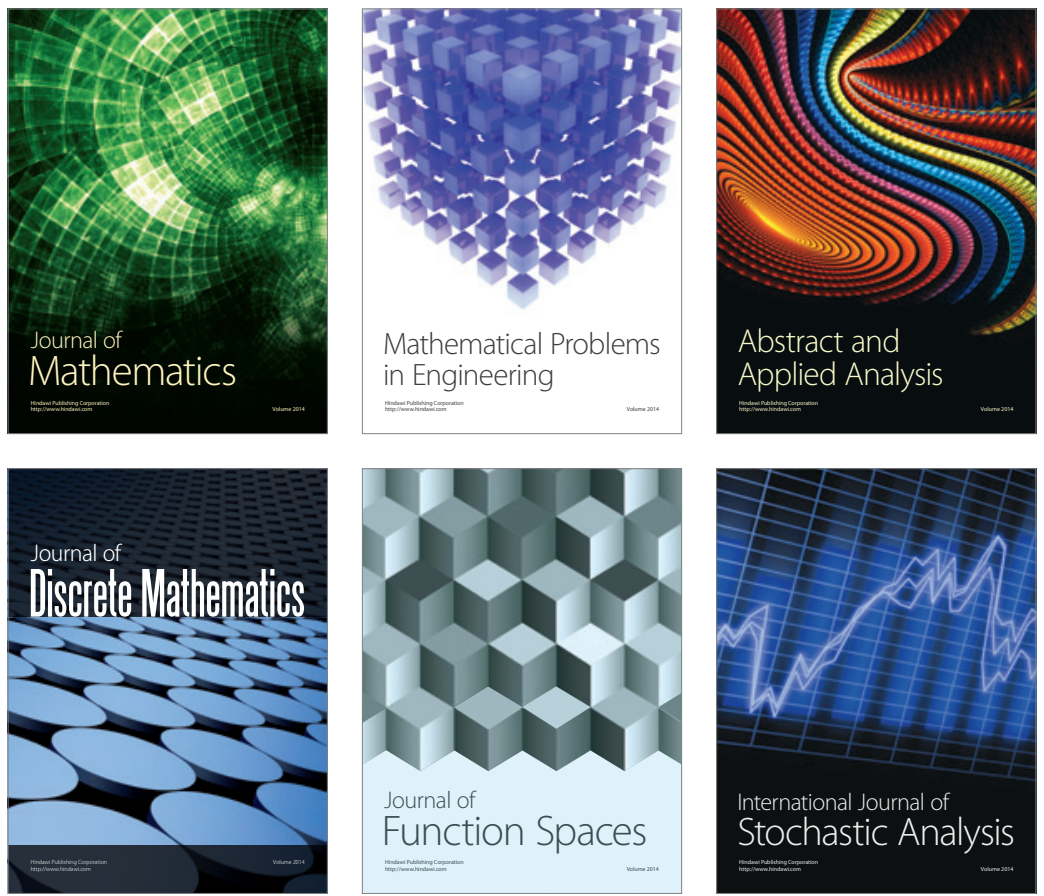

Journal of

Function Spaces

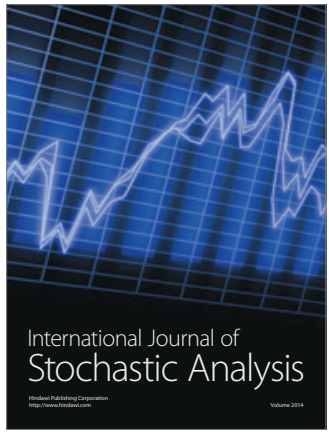

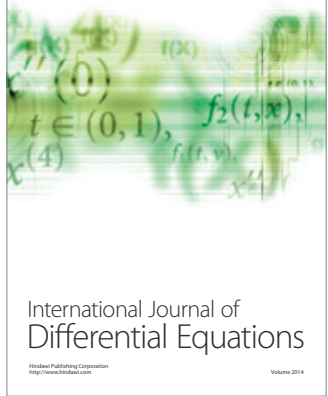
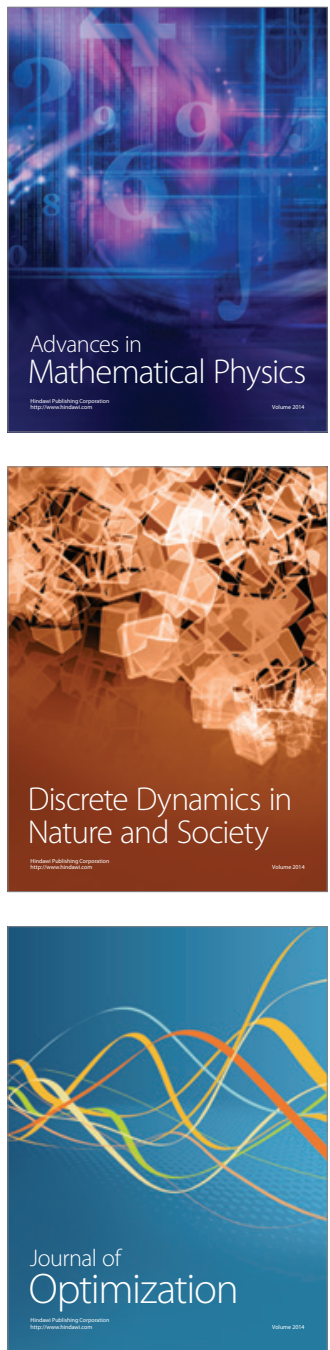